Research Paper

\title{
Increased Expression of Macrophage Migration Inhibitory Factor and DJ-I contribute to Cell Invasion and Metastasis of Nasopharyngeal Carcinoma
}

\author{
Xiao-juan PEI ${ }^{1}$, Tong-tong WU ${ }^{1}$, Bin LI 2 ${ }^{2}$ Xiao-ying TIAN ${ }^{3}$, Zhi LI ${ }^{2}$, Qing-xu YANG ${ }^{1}$ 冈 \\ 1. Department of Pathology, Huizhou Municipal Central Hospital, 41 Eling Road North, Huizhou 516001, China \\ 2. Department of pathology, the First Affiliated Hospital, Sun Yat-sen University, 58 Zhongshan Road II, Guangzhou 510080, China \\ 3. School of Chinese Medicine, Hong Kong Baptist University, 7 Baptist University Road, Kowloon Tong, Hong Kong, China
}

$\square$ Corresponding author: Dr. Qing-xu YANG, Department of Pathology, Huizhou Municipal Central Hospital, 41, Eling Road North, Huizhou 516001, China. Tel: (86)752 2288244; E-mail: hzbingli@126.com

( ) Ivyspring International Publisher. This is an open-access article distributed under the terms of the Creative Commons License (http://creativecommons.org/ licenses/by-nc-nd/3.0/). Reproduction is permitted for personal, noncommercial use, provided that the article is in whole, unmodified, and properly cited.

Received: 2013.07.26; Accepted: 2013.12.09; Published: 2014.01.0I

\begin{abstract}
Background and aim: Both macrophage migration inhibitory factor (MIF) and DJ-I protein have been shown to relate with cell invasion and metastasis in tumors. However, the role of $D J-I$ in invasion and metastasis of nasopharyngeal carcinoma (NPC) and its relation to MIF expression in NPC are not fully understood. The aim of present study is to determine whether or not MIF and DJ-I are correlated with tumor invasion and influence a worse outcome in NPC, as well as its related mechanism.

Methods: 125 cases of NPC and 45 normal tissues of nasopharynx were collected. The expression of MIF and DJ-I in tissue microarray was evaluated by immunohistochemical staining. Correlation between immunostainings and clinicopathological parameters, as well as the follow-up data of patients, was analyzed statistically. The association of MIF and DJ-I with cell invasion and migration in NPC cell line were evaluated by small interfering RNA (siRNA) transfection, invasion assay and Western blotting.

Results: MIF and DJ-I staining was diffused and strong in tumor cells, whereas they were generally weaker and less common in normal lining epithelia of nasopharynx. High MIF expression in tumor cells $(7 \mid .2 \%, 89 / 125$ cases) were significantly associated with advanced clinical stage, lymph node metastasis, and worse prognosis of NPC patients. High expression of DJ-I (75.2\%, 94/I 25 cases) were closely correlated to lymph node metastasis and MIF high-expression. Only MIF high expression $(P=0.010)$ and lymph node metastasis $(P=0.004)$ emerged as strong independent prognostic factors for overall survival of NPC patients. In vitro, down-regulated expression of DJ-I in NPC cell lines by siRNA was observed to reduce cell migration and invasion potential, however, exogenous MIF promoted cells invasion.

Conclusions: The data provided evidence that increased expression of MIF and DJ-I induced cell invasion and metastasis of NPC, supporting the idea that MIF and DJ-I may play important roles as regulators in the progression of NPC.
\end{abstract}

Key words: nasopharyngeal carcinoma (NPC); macrophage migration inhibitory factor (MIF); DJ-1; invasion and metastasis; prognosis

\section{Introduction}

Nasopharyngeal carcinoma (NPC) is an Epstein-Barr virus (EBV)-associated malignant tumor that has a high incidence in southern China, Southeast
Asia, Alaska, and North Africa [1-2]. Despite substantial improvements in treatment for NPC patients in recent decades, the 5-year overall survival rate is 
only $50-60 \%$ because of the frequency of distant metastasis and local recurrence [3-4]. Unlike other head and neck cancer, NPC tumor cells are likely to invade to the surrounding tissue or metastasize to lymph nodes in the early stage of tumor development [5], which is the major cause for advanced clinical stage and poor prognosis of this tumor. In clinical practice, the early symptoms of this tumor are usually quite non-specific, over $75 \%$ of NPC patients present with late stage disease when it is being diagnosed [6]. Although various studies on NPC have been performed recently to try to identify the mechanism of invasion and metastasis, the precise molecular mechanism remains to be clarified. In fact, to date, none has been proven to be sufficiently reliable to be of practical use clinically.

Macrophage migration inhibitory factor (MIF) is a multi-functional cytokine, which is associated with inflammation and tumorigenesis [7]. Current studies have shown that MIF is over-expressed in a variety of human tumors, including those of the colon, prostate, breast, lung, and liver, as well as in melanoma and glioblastoma multiforme [8-14]. It has also been indicated that MIF modulates malignant transformation and promotes tumor cell invasion, proliferation, angiogenesis, and metastasis [15-16]. Various studies have suggested that MIF can influence the cellular biological response by inducing the nuclear factor-kappa B (NF- kB), Erk1/2 and activating protein-1 (AP-1) signaling pathways, or by binding CD74/CD44 receptor complex [17]. MIF-induced activation of PI3K/Akt pathway and $\alpha_{v} \beta_{3}$ integrin has been also demonstrated to involve in the development and progression of breast, gastric cancer and chondrosarcoma [18-20]. Recently, tumor-derived MIF has been shown to closely correlate with tumor aggressiveness and metastatic potential. The high expression of MIF in tumor is considered to be a poor prognostic factor in patients with lung adenocarcinoma, breast, and hepatocellular carcinomas. In NPC, our previous study demonstrated that increased expression of MIF in neoplastic cells was closely in relation to higher expression of matrix metalloproteinases-2, -9 and lymph node metastasis of NPC [21]. MIF may also contribute to angiogenesis by inducing upregulation of IL-8 expression in an autocrine EBV-independent pathway [22]. However, whether or not MIF as an independent factor for predicting the prognosis of NPC patients remains to be investigated.

DJ-1 gene is a mitogen dependence oncogene, which maps at chromosome 1p 36.2-p36.3, and first identified to synergically transform mouse NIH 3T3 cells with activated ras [23]. Early study showed that the mutation of DJ-1 gene might cause Parkinsonism disease [24]. Subsequent studies have shown that DJ-1 is a ubiquitous and highly conserved protein that affects cell death, proliferation, and cell cycle progression exhibiting closely relationship to the development, progression and prognosis of the malignant tumors. Recent studies have demonstrated that DJ-1 expression is increased in breast cancer, lung cancer, prostate cancer and ovarian cancer [25-27]. DJ-1 has also been shown to promote the invasion and metastasis of tumor cell and to be a prognostic factor predicting poor outcome in cancers patients [28-30]. So far, to our knowledge, there is no study to investigate the role of DJ-1 in the development and progression of NPCs.

Previous studies have shown that MIF modulates cell migration and invasion in human cancer cells, including NPC. Although both MIF and DJ-1might play their roles in modulating tumor cells biological behavior via some universal pathways, such as Erk, JNK MAPK and the PI3K signaling pathways, their effect on invasion and metastasis in NPC are mostly unknown. In this study, the expression of DJ-1 in NPC was explored and the correlation between MIF and DJ-1 in NPCs was evaluated for the first time. In addition, the role of the DJ-1 on invasion and migration in NPC cells was also investigated by transfecting the specific siRNA in NPC cells in vitro. We postulated that there exist some potential links between MIF and DJ-1 in the progression of NPC.

\section{Methods}

\section{Specimens of NPC and normal nasopharyngeal tissue}

Archival formalin-fixed, paraffin-embedded specimens from 125 primary NPC patients before treatment during 2004-2010 in the first Affiliated Hospital of Sun Yat-sen University and Huizhou Municipal Central Hospital were collected. There were 85 males and 40 females with a median age of 51 years (range 23-77). According to World Health Organization (WHO) histological classification, 119 patients were diagnosed as undifferentiated non-keratinized carcinoma (UNKC) and 6 were diagnosed as differentiated non-keratinized carcinoma (DNKC). The patients were classified the disease stage according to the criteria of China NPC 92 staging system [31], and 5 in stage I, 37 in stage II, 51 in stage III, and 32 in stage IV. All the patients were treated with standard curative radiotherapy with or without chemotherapy. Forty five normal tissues of nasopharynx were also collected as healthy control from individuals undergoing NPC screening in the first Affiliated Hospital, Sun Yat-sen University in the same period. 


\section{Paraffin tissue microarray construction}

In this study, the paraffin tissue microarray construction was conducted by previous description [32]. Briefly, section was cut from each paraffin block and stained with hematoxylin and eosin (H\&E). Each donor block was overlaid with the corresponding H\&E slide and observed by experienced pathologists. The area in donor block for tissue microarray sampling was verified according to the H\&E slide and marked. A manual tissue arrayer (Beecher Instruments, Inc., Sun Prairie, WI) was used for array construction. Two representative $1.0 \mathrm{~mm}$ cores were removed from each donor block and transferred to pre-molded recipient paraffin block with designed orientation. An additional 6 cores derived from tonsil, lymph node and breast carcinoma were used as control materials. The serial sections with 4-mm thickness were cut from arrayed block and mounted on APES-coated glass slides and stored at $4^{\circ} \mathrm{C}$ for further detection.

\section{Immunohistochemistry and scoring}

The sections were subjected to immunostaining using a ChemMate Envision/HRP Kit (DAKO Co., Glostrup, Denmark). Slides were deparaffinized in xylene and rehydrated in decreasing concentrations of ethanol and rinsed in phosphate buffered saline. After blocking with normal serum for $10 \mathrm{~min}$, the slides were incubated with a 1:100 dilution of mouse monoclonal MIF antibody (Abcam, Cambridge, MA), and a 1:200 dilution of the goat anti-human polyclonal DJ-1 antibody (Santa Cruz Biotechnology, Santa Cruz, CA) for $60 \mathrm{~min}$, respectively. Slides were detected by ChemMate Envision/HRP Kit for 30min at room temperature and followed by developing with diaminobenzidine (DAB) for visualization. Negative controls were carried out by substituting non-immune serum for the primary antibodies. The immunostaining results were evaluated and scored semiquantitatively by two pathologists without knowledge of the clinical data of patients. Evaluation of the MIF and DJ-1 expression was performed by stain intensity and stain area (double scoring system) as previously described [22, 30]: (score 0), no staining; (score 1), weak staining; (score 2), moderate staining; (score 3), strong staining. Staining area less than 30\% (score 1); $31-75 \%$ (score 2); more than $75 \%$ (score 3 ) of tumor cells. The high-expression of MIF and DJ-1 was defined when immunostaining score was equal or more than 4 (stain intensity score times stain area score), whereas the other cases were grouped into the low-expression category.

\section{Cell culture and reagents}

In this study, the human NPC cell lines CNE-1 and CNE-2 were used. Both cell lines were stored in the Department of pathology, the first affiliated hospital, Sun Yat-sen University and maintained in Dulbecco's modified Eagle's medium (DMEM) culture medium with $10 \%$ heat-inactivated fetal calf serum (FCS) and antibiotics $(50 \mathrm{U} / \mathrm{ml}$ penicillin and 100 $\mathrm{ug} / \mathrm{ml}$ streptomycin, Invitrogen, Carlsbad, CA) at $37^{\circ} \mathrm{C}$ in a humidified incubator with $5 \% \mathrm{CO}_{2}$. Human recombined MIF (Abnova corporation, Taiwan) was dissolved in culture medium for in vitro experiments.

\section{Small interfering RNA (siRNA) targeting DJ-I}

Human DJ-1 siRNAs (si DJ-1) and its unrelated siRNA used as negative control (si Control) were designed and synthesized by Ribo Biotechnology Co. (Beijing, China). Three pairs of DJ-specific siRNA were designed in this study. si RNA 1 targeted GATTAAGGTCACCGTTGCA, RNA2 targeted GAATTTATCTGAGTCTGCT, and si RNA3 targeted TGATGAATGGAGGTCATTA. However, in current study, only si RNA 2 was used for in vitro study because it worked well in preliminary experiment. The siRNA sequence: positive-sense strand, 5'-GAAUUUAUCUGAGUCUGCU-3'; antisense strand, 3'-CUUAAAUAGACUCAGACGA-5'. si Control: TTCTCCGAACGTGTCACGT (positive-sense strand, 5'-UUCUCCGAACGUGUCACGU-3'; antisense strand, 3'-AAGAGGCUUGCACAGUGCA-5'). siRNAs were reconstituted, and subsequent transfections were conducted in six-well plates using TurboFect siRNA Transfection reagent (Fermentas, Maryland, USA), according to the manufacturer's instructions. In brief, CNE-1 and CNE-2 cells were seeded into six-well plates with a density of $2 \times 10^{5}$ cells/well. Once the cells reached $80 \%$ confluence, they were treated with either specific siRNA or siControl (50 nM) complexed with TurboFect. Ten percent FCS was added $4 \mathrm{~h}$ after transfection, and fresh DMEM with $10 \%$ FBS was added as needed after $24 \mathrm{~h}$. Cells were collected at $48 \mathrm{~h}$ after transfection to assess DJ-1 protein levels by Western blot and the other functional assays listed in the following sections.

\section{Western blotting assay}

CNE-1 and CNE-2 cells were cultured in DMEM medium with $10 \%$ FCS until $70 \%$ cell confluence. Medium was changed to DMEM with 1\% FCS (v/v), and the cells were treated with final concentration of $25 \mathrm{ng} / \mathrm{ml} \mathrm{MIF}$ for $48 \mathrm{~h}$. After treatment with MIF, cell lysates were prepared as previously described [33]. si DJ-1transfected CNE-1 and CNE-2 cells were also lysed. The equal protein samples were subjected to $12 \%$ SDS-PAGE electrophoresis, followed by the transfer to a polyvinylidene fluoride (PVDF) membrane, blocking in 5\% fat-free milk and incubation with MIF (Abcam, 1:500 dilution), DJ-1 (Santa Cruz 
Bio., 1:1000 dilution), Akt (Abcam, 1:500 dilution), pAkt (Abcam, 1:500 dilution) or Actin antibody (Abcam, Cambridge, MA ) at $4^{\circ} \mathrm{C}$ overnight. Detection was performed using horseradish peroxidase-conjugated secondary antibody and enhanced chemiluminescence reagents from Amersham (Amersham Life Sciences, UK). The relative optical density (ROD, ratio to Actin) of each blot band was quantified by NIH Image software (Image J 1.36b).

\section{Immunofluorescence staining}

MIF-treated or si DJ-1transfected CNE-1 and CNE-2 cells were plated on coverslips, fixed with $4 \%$ paraformaldehyde/phosphate-buffered saline (PBS) for $15 \mathrm{~min}$ and permeabilized with $0.1 \%$ Triton X-100 in PBS for $2 \mathrm{~min}$, and then incubated in PBS containing $5 \%$ skim milk for $1 \mathrm{~h}$ at room temperature. Cells were incubated with anti-MIF or anti-DJ-1 antibody for $1 \mathrm{~h}$ at room temperature, followed by incubation with IgG/Cy3 (1:500, Invitrogen, Carlsbad, CA) for 1h and nuclear counter staining with DAPI.

\section{In vitro wound healing assay}

Migratory ability of NPC cells was measured using the in vitro wound healing assay. Cells were seeded in six-well plates and transfected with specific siRNAs or siControl for $48 \mathrm{~h}$. MIF-treated or si DJ-1transfected cells were grown to $100 \%$ confluence. Wounds were created by scraping monolayer cells with a sterile pipette tip. At 0,12 , and $24 \mathrm{~h}$ after the creation of wounds, wound distances were measured at each time point and expressed as the average percent of wound closure by comparing the zero time.

\section{Cell invasion assay}

Tumor cell invasion was assayed by Transwell chamber (Becton Dickinson, Franklin Lakes, NJ) with 8-um-porosity polycarbonate filter membrane. The upper layers of membranes were precoated with $30 \mathrm{ul}$ Matrigel matrix, a material that mimics the basement membrane, containing 10\% DMEM, 5\% 10×HEPES, $35 \%$ acidic collagen (rat tail), and 50\% Matrigel in each Transwell chamber. MIF-treated or si DJ-1 transfected CNE- 1 and CNE- 2 cells (500ul, $1 \times 10^{5} \mathrm{cell} / \mathrm{ml}$ medium with $1 \%$ FCS) were placed into the upper side of the coated and rehydrated cell chamber, respectively. This chamber was inserted into each well of a 24-well plate filled with $500 \mathrm{ul} \mathrm{medium} \mathrm{(DMEM} \mathrm{with} 5 \%$ FCS). The cells in the chamber were incubated for another $24 \mathrm{~h}$ at $37^{\circ} \mathrm{C}$, and the cells which went through the coated membrane to the underside of the filter were counterstained by $1 \%$ crystal violet to reveal the nuclei. For each chamber, the number of invasive tumor cells was counted in five random high-power fields (HPF; 10×40) by reversed light microscopy.

\section{Statistical Analysis}

All statistical analysis was carried out by using SPSS 13.0 software for Windows. Chi-square test was used to assess MIF and DJ-1 expression with respect to clinicopathological characteristics. The survival curve of patients was determined by Kaplan-Meier method and Cox regression, and statistical evaluation was performed using the log-rank test. Results derived from in vitro study were presented as mean \pm standard deviation (SD). Data were analyzed by one-way analysis of variance (ANOVA) with Dunnett's post hoc test and Turkey's post hoc test for multigroup comparisons. A $\mathrm{P}$ value $<0.05$ was considered statistically significant.

\section{Results}

\section{MIF and DJ-I expression in normal tissue of nasopharynx and NPCs}

Both MIF and DJ-1 positive staining signals were observed within the cytoplasm of cells. MIF and DJ-1 staining was diffused and strong in tumor cells, whereas they were generally weaker and less common in normal lining epithelia of nasopharynx. A significant difference in expression level of DJ-1 or MIF was demonstrated between NPC and normal tissues (both $\mathrm{P}<0.001$ ) (Figure 1a-d). Among 125 NPC cases, MIF high-expression was observed in $71.2 \%$ $(89 / 125)$ of NPC tissues. High expression of MIF was significantly associated with the advanced clinical stage (stage III and IV) $(\mathrm{P}=0.002)$ and lymph node metastasis $(\mathrm{P}=0.006)$. $75.2 \%(94 / 125)$ of NPCs were assessed as high-expression of DJ-1, and only lymph node metastasis was observed to link to DJ-1 high-expression $(\mathrm{P}=0.002)$. There was not any significant association between overall DJ-1 expression and patient age, gender, and tumor stage. Furthermore, high expression of DJ-1 exhibited a close correlation with high expression of MIF in tumor cells (Table 1).

\section{High expression of MIF, but not DJ-I was associated with poor prognosis of NPC patients}

In this study, 92 cases were followed up from 3 to 60 months with mean period of 32.5 months, whereas 33 cases were excluded from survival analysis because the patients were not to be contacted during the period of follow-up. The overall survival rate of patients was $64.1 \%$, and 33 patients had died of tumor in this period. The survival rates of patients with early clinical stage (stage I-II) and advanced stage (stage III-IV) were $76.4 \%$ and $56.8 \%$, respectively. In univariate survival analyses, we analyzed the impact of all of the clinicopathological parameters on overall survival. The lymph nodal status and MIF 
expression level reached significance for overall survival (Table 1). Figure 1e and 1f illustrate the impact of lymph nodal status and MIF expression on survival times. In multivariate analysis, we found that the overall survival time was significantly dependent on lymph node metastasis, clinical stage and MIF expression level in tumor cells. However, DJ-1 expression was not associated with the survival time of patients (Table 2).
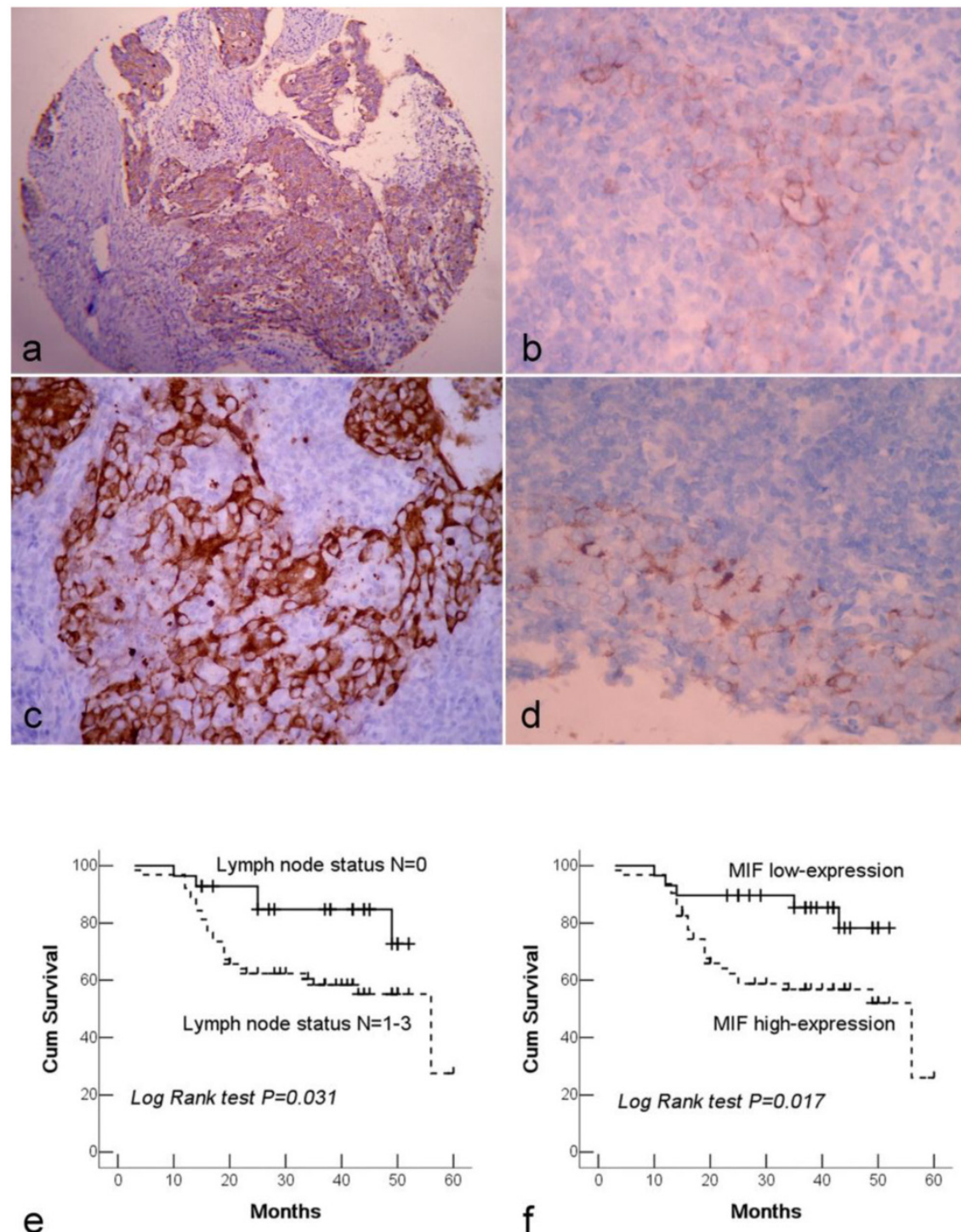

Figure I. Immunohistochemical staining of NPC tissue microarray and their correlation with survival of NPC patients. (a). High expression of MIF shown under lower power field in the microarry section. (b). Cytoplasmic weak staining of MIF in lining epithelium of nasopharynx. (c). High expression of DJ-I showed positive immunostaining signal was localized in the cytoplasm of tumor cells. (d). The DJ-I staining in normal epithelia of nasopharynx was weaker and local. (e). By Kaplan-Meier survival analysis, patients with lymph node metastasis had a lower overall survival rate than those without lymph node metastasis. (f). There was a significant difference in overall survival rate between the patients with MIF high-expression and low-expression. (a: Immunohistochemical staining with original magnification, I00x; b-d: Immunohistochemical staining with original magnification, $400 \times$ ) 

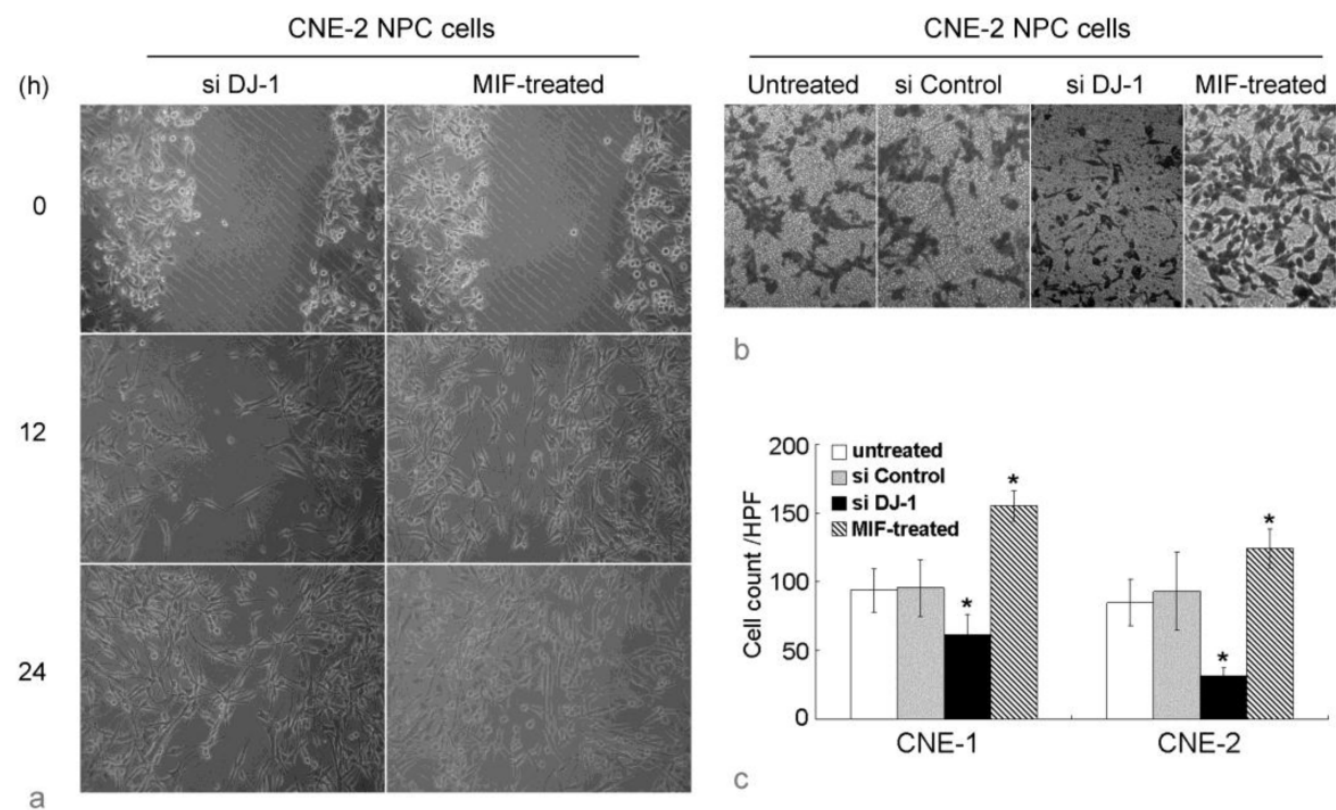

b

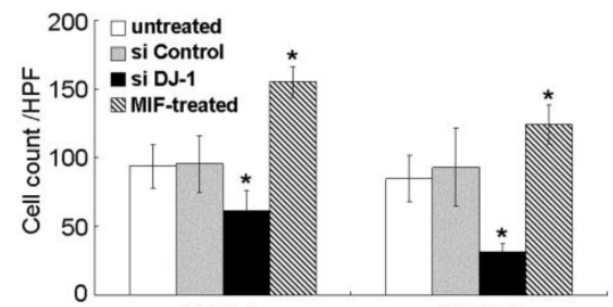

CNE-1

CNE-2

Figure 2. MIF and DJ-I regulate NPC cell migration and invasion. (a). Optical microscopic images of in vitro wound healing at 0,12 , and $36 \mathrm{~h}$ after the creation of wounds in NPC cells. The decreased cell migration in CNE-2 NPC cells by si DJ-I was observed. However, exogenous MIF-treated NPC cells displayed significantly faster wound closure at all time points. (b). In invasion assay using Transwell chamber, the count of cells which had passed through the 8-um-pore membrane and attached to the undersurface was almost equal in untreated and si Control-transfected CNE-2 NPC cells. However, in si DJ-I-transfected CNE-2 NPC cells, the passed cells was obviously decreased. MIF-treated CNE-2 cells showed significantly increased invasive ability. (c). Cell count evaluated in CNE-I and CNE-2 NPC cells with or without treatment. *, Significant difference in cell count compared with untreated or si Control-transfected cells.

Table I. Correlation between proteins expression and clinicopathological parameters of NPC patients

\begin{tabular}{|c|c|c|c|c|c|}
\hline \multirow[t]{2}{*}{ Variable } & \multicolumn{2}{|c|}{ MIF expression a } & \multicolumn{2}{|c|}{ DJ-1 expression a } & \multirow[t]{2}{*}{ Survival time (months) b } \\
\hline & Low $(n=36)$ & High $(n=89)$ & Low $(n=31)$ & High $(\mathrm{n}=94)$ & \\
\hline \multicolumn{6}{|l|}{ Age (year) } \\
\hline$<51(\mathrm{n}=61)$ & 19 & 42 & 14 & 47 & $44(39-49)$ \\
\hline \multirow{2}{*}{$\geq 51(\mathrm{n}=64)$} & 17 & 47 & 17 & 47 & $41(35-48)$ \\
\hline & \multicolumn{2}{|c|}{$\mathrm{P}=0.599$} & \multicolumn{2}{|c|}{$\mathrm{P}=0.572$} & $\mathrm{P}=0.296$ \\
\hline \multicolumn{6}{|l|}{ Gender } \\
\hline Male $(n=85)$ & 26 & 59 & 18 & 67 & $42(37-47)$ \\
\hline \multirow{2}{*}{ Female $(\mathrm{n}=40)$} & 10 & 30 & 13 & 27 & $40(35-46)$ \\
\hline & \multicolumn{2}{|c|}{$\mathrm{P}=0.423$} & \multicolumn{2}{|c|}{$\mathrm{P}=0.102$} & $\mathrm{P}=0.460$ \\
\hline \multicolumn{6}{|l|}{ WHO Histotype } \\
\hline $\operatorname{DNKC}(\mathrm{n}=6)$ & 2 & 4 & 2 & 4 & $43(39-48)$ \\
\hline \multirow[t]{2}{*}{ UNKC $(n=119)$} & 34 & 85 & 29 & 90 & $32(15-49)$ \\
\hline & \multicolumn{2}{|c|}{$\mathrm{P}=0.611$} & \multicolumn{2}{|c|}{$\mathrm{P}=0.233$} & $\mathrm{P}=0.506$ \\
\hline \multicolumn{6}{|l|}{ Tumor size } \\
\hline $\mathrm{T} 1-2(\mathrm{n}=55)$ & 13 & 42 & 13 & 42 & $44(39-50)$ \\
\hline \multirow[t]{2}{*}{ T3-4 $(n=70)$} & 23 & 47 & 18 & 52 & $37(31-42)$ \\
\hline & \multicolumn{2}{|c|}{$\mathrm{P}=0.233$} & \multicolumn{2}{|c|}{$\mathrm{P}=0.777$} & $\mathrm{P}=0.471$ \\
\hline \multicolumn{6}{|c|}{ Lymph node metastasis } \\
\hline No $(n=33)$ & 15 & 18 & 14 & 19 & $46(41-51)$ \\
\hline \multirow[t]{2}{*}{$\mathrm{N} 1-3(\mathrm{n}=92)$} & 21 & 71 & 17 & 75 & $40(35-45)$ \\
\hline & \multicolumn{2}{|c|}{$\mathrm{P}=0.006$} & \multicolumn{2}{|c|}{$\mathrm{P}=0.002$} & $\mathrm{P}=0.031$ \\
\hline \multicolumn{6}{|l|}{ Clinical stage } \\
\hline Stage I-II $(\mathrm{n}=42)$ & 19 & 23 & 13 & 29 & $43(38-49)$ \\
\hline \multirow[t]{2}{*}{ Stage III-IV $(n=83)$} & 17 & 66 & 18 & 65 & $41(35-46)$ \\
\hline & \multicolumn{2}{|c|}{$\mathrm{P}=0.002$} & \multicolumn{2}{|c|}{$\mathrm{P}=0.216$} & $\mathrm{P}=0.113$ \\
\hline \multicolumn{6}{|l|}{ MIF expression } \\
\hline Low $(n=36)$ & & & 20 & 16 & $46(41-51)$ \\
\hline High $(n=89)$ & & & 11 & 78 & $39(34-45)$ \\
\hline & & & & & $\mathrm{P}=0.017$ \\
\hline DJ-1 expression & & & & & \\
\hline Low $(n=31)$ & 20 & 11 & & & $43(38-48)$ \\
\hline $\operatorname{High}(\mathrm{n}=94)$ & 16 & 78 & & & $42(37-47)$ \\
\hline & & & & & $\mathrm{P}=0.290$ \\
\hline
\end{tabular}


Table 2. Cox regression model for multivariate analyses of prognostic factor in NPC

\begin{tabular}{|c|c|c|c|}
\hline Variable & Hazard ratio & 95\% confidence interval & $P$ value \\
\hline Age (<51 vs. $\geq 51)$ & 1.713 & $0.803-3.655$ & 0.163 \\
\hline Gender (male vs. female) & 0.803 & $0.335-1.925$ & 0.623 \\
\hline WHO Histotype (DNKC vs. UNKC) & 0.316 & $0.066-1.510$ & 0.149 \\
\hline Tumor size $\left(\mathrm{T}_{1-2}\right.$ vs. $\left.\mathrm{T}_{3-4}\right)$ & 0.726 & $0.348-1.517$ & 0.395 \\
\hline Lymph node metastasis ( $\mathrm{N}_{0}$ vs. $\mathrm{N}_{1-3}$ ) & 10.503 & $2.089-52.787$ & 0.004 \\
\hline Clinical stage (Stage I-II vs. III-IV) & 0.240 & $0.059-0.971$ & 0.045 \\
\hline MIF expression (low vs. high) & 5.136 & $1.464-18.008$ & 0.010 \\
\hline DJ-1 expression (low vs. high) & 0.662 & $0.224-1.959$ & 0.457 \\
\hline
\end{tabular}

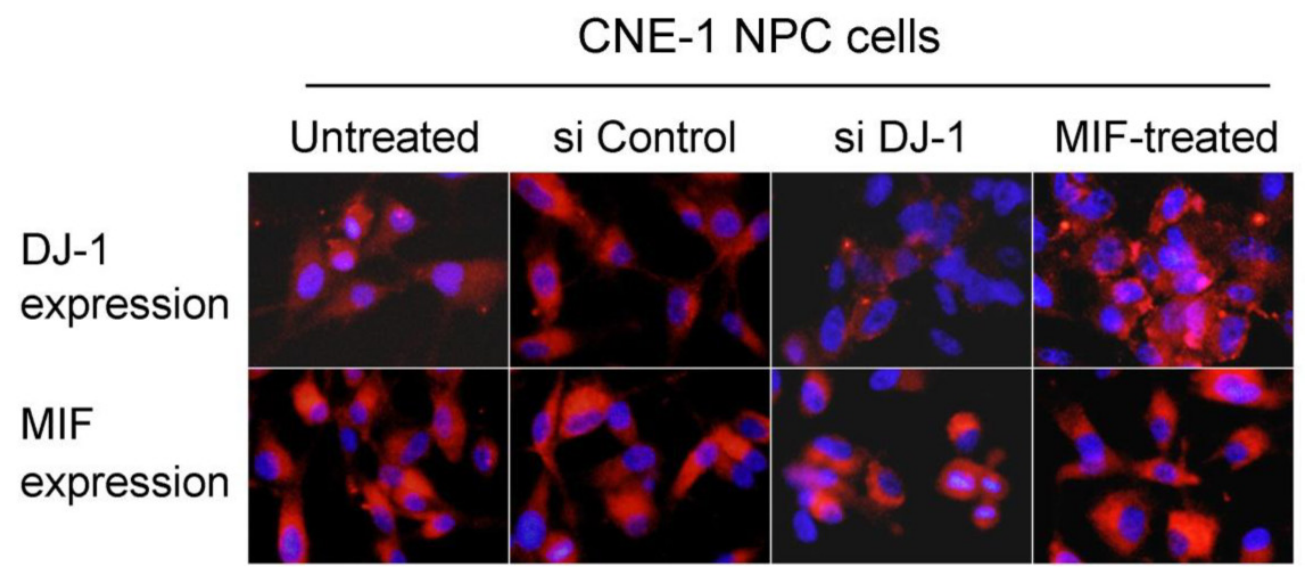

Figure 3. MIF and DJ-I expression in NPC cells in vitro. Immunofluorescence assay showed that DJ-I protein expression was significantly decreased in CNE-I NPC cells with si DJ-I transfection when compared with untreated or control siRNA transfected cells. However, DJ-I expression level was observed to increase in MIF-treated NPC cells. MIF expression level was stable in CNE-I NPC cells regardless of treatment (Immunofluorescence staining with original magnification, 400x).

\section{MIF altered the expression of DJ-I and pAkt level in NPC cells in vitro}

MIF and DJ-1 could be detected in the cytoplasm of CNE-1 and CNE-2 NPC cells by immunofluorescence staining. After treatment by MIF for $48 \mathrm{~h}$, the DJ-1 protein level of NPC cells was significantly increased than those without MIF treatment (Figure 3). We determined whether DJ-1 was capable of regulating migration and invasion via the Akt pathway in these NPC cells. Silencing of DJ-1 reduced phosphorylation of Akt, but not total Akt in NPC cells. In addition, the level of pAkt was found to be increased in tumor cells with MIF treatment (Figure 4). These results indicated that both MIF and DJ-1 might exert their influence in NPCs through the Akt pathway.

\section{Discussion}

Lymphatic spread in NPCs is an important early event. The current study exhibited that lymph node status is a strong independent prognostic factor of NPC. The result of relationship between MIF high expression and lymph node metastasis, as well as advanced clinical stage of tumor indicated that MIF might be a possible mediator to contribute to the extensive lymph node metastasis and accelerate the poor progression of NPC. High expression of MIF was closely in relation to lower survival rate of NPC pa- tients either on univariate analysis or on multivariate analysis. These results indicate that MIF alone may be an independent prognostic factor for patients with NPC. These findings were consistent with the role of MIF in prognostic prediction in hepatocellular carcinoma, colon cancer, and prostate cancer [34-36]. In those studies, high expression of MIF is a valuable marker for prognostic prediction in tumors. The association of cell invasion and migration with lymphatic metastasis has been investigated in many malignant tumors. The numerous contributions of cell invasion and migration during metastasis such as matrix metalloproteinase (MMPs), CD44 and several angiogenic molecules, including VEGF, IL-8 to the metastatic potential of tumors have been evaluated [37-38]. In the present study, exogenous MIF treatment resulted in significantly increased cell migration and invasion of NPC cells in vitro. It suggests that MIF is likely to be a local mediator though autocrine or paracrine regulatory loop to promote cell invasion $[18,39]$. Although the current examinations in this study were not sufficient to allow us to make definite conclusion, these results indicated that MIF may contribute to lymph node metastasis in NPC through the enhancement of cell migration and invasion. MIF-related cell invasion may play some important roles in NPC progression and influence the prognosis of NPC patients. Thus, we consider that the meas- 
urement of MIF in tumor cells of NPC is able to be a valuable clinical parameter to directly predict the prognosis of NPC patients, because it exhibits closely correlation to lymph node status and clinical stage.
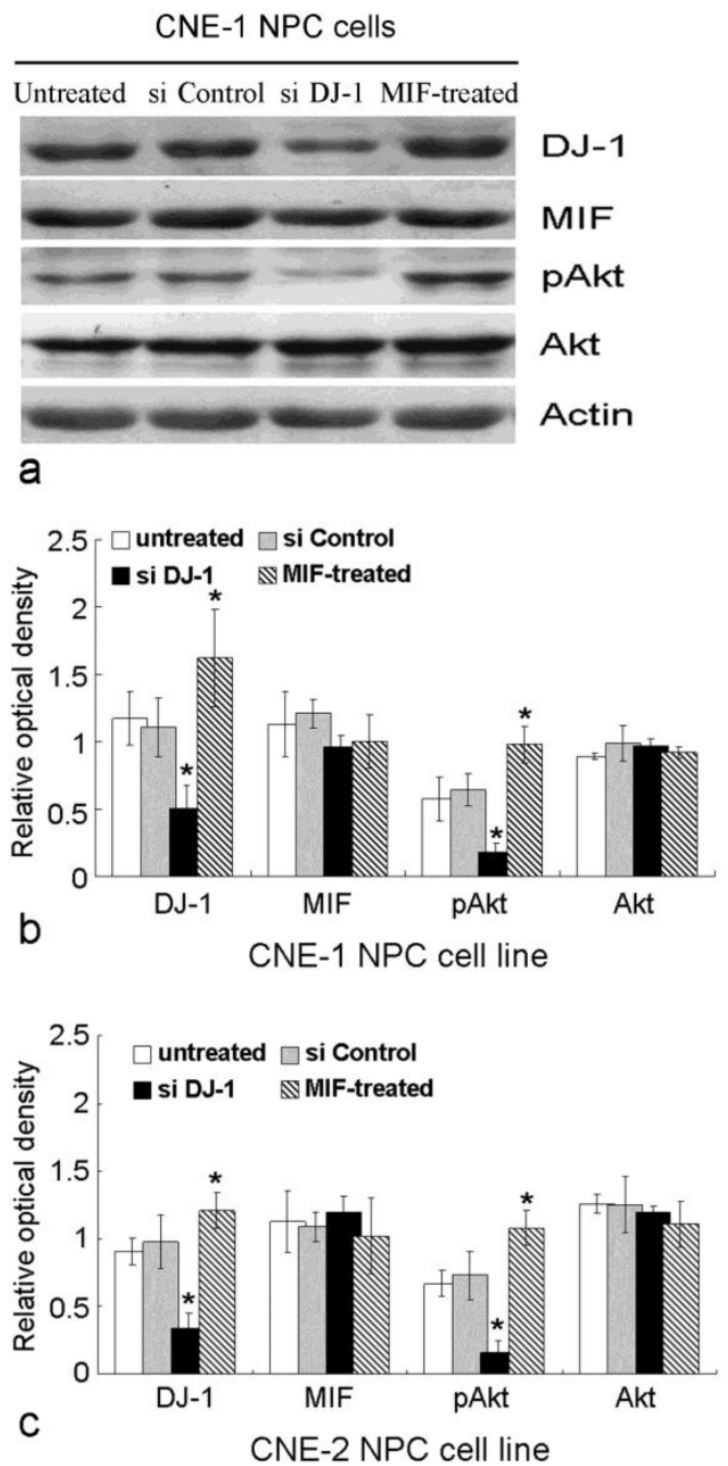

Figure 4. MIF and DJ-I regulate the expression of Akt. (a). Western blot assay exhibited that the expression of DJ-I and pAkt in CNE-I NPC cell was significantly decreased by si-DJ-I treatment. However, after MIF treatment, CNE-I NPC cells showed increased expression of DJ-I and pAkt. (b) and (c) showed significantly altered expression of DJ-I and pAkt in CNE-I and CNE-2 cells, respectively. *, Significant difference in expression level compared with untreated or si Control-transfected cells.

As a mitogen dependent oncogene, DJ-1 has been identified to play some important roles in various physiological and pathophysiological processes. DJ-1 can be induced by oxidative stress and is an important mediator of the hypoxia-induced cellular responses [40]. The expression of DJ-1 has been found to be increased in the serum, effusion and tumor samples of patients with cancer of the breast, lung, ovary, and esophagus $[25,27,41]$. The measurement of DJ-1 antibodies in peripheral blood was thought be a use- ful marker of detection the occurrence and development of breast cancer [42]. DJ-1 has been shown to be a prognostic factor predicting poor outcome in cancers patients $[30,43]$. The recurrence rate of patients with high-level of DJ-1 obviously was higher than that with low-level of DJ-1. Positive relationship of DJ-1 with tumor invasion and metastasis was also found in various cancers, including hepatocellular carcinoma, glioma and non-small cell lung cancer [29, 44-45]. In the present study, we found that high-expressed DJ-1 protein was closely associated with lymph node metastasis of NPC patients. Concurring in vitro study demonstrated that significantly decreased cell migration and invasion of NPC cells was due to the interference of DJ-1 protein. These results provided evidence of effect of DJ-1 on NPC progression by the way of promoting lymph node metastasis, and at least, partly through enhancing the cell invasion and migration. However, the high level of DJ-1 expression might not indicate advanced clinical stage of patient, and unexpectedly, we did not find any associations of DJ-1 expression with prognosis of NPCs. Thus, we did not find evidence of the role of DJ-1 in prognostic prediction of NPCs although its expression level was indeed in relation to lymph node metastasis. We speculate that the relevance of DJ-1 in NPCs may be limited and locally. Of course, further studies are clearly necessary to clarify the involved mechanisms in larger cohorts.

At the present study, we found that DJ-1 high expression in NPCs was significantly correlated with MIF high expression. Exogenous MIF treatment in NPC cells in vitro was found to induce up-regulation of DJ-1 protein. Although DJ-1 protein level in tumor cells was not an independent predictive factor for prognosis of NPCs, it should be considered to take part in the development and progression of NPC. Recently, increasing evidence has suggested that the function of DJ-1 was associated with Akt activation or ERK/uPA pathway [28, 46]. Down-regulation of DJ-1 inhibits endogenous Akt phosphorylation in cancer cell lines [46-47]. Accumulated researches also show MIF can modulate cell biological behaviors by activating the NF-kB, Erk1/2, AP-1, and PI3K/Akt pathway, which is similar to the DJ-1 mechanism. In the present study, the results showed that both MIF and DJ-1 might exert their influence in NPC cells through the Akt pathway in vitro, and exogenous MIF induced up-regulated expression of DJ-1 in tumor cells. We postulate that there exist some potential interaction between MIF and DJ-1 in tumor cell, but the precise mechanism involved is still to be investigated further. The lymphatic metastasis in NPC may be a complex process, and multiple induce factors, including MIF and DJ-1 involved in this process 
through their own specific pathways. Just for these reasons, high expression of MIF and DJ-1 might be associated with lymph node metastasis of NPC; however, a single molecule expression status in tumor might not be a strong independent factor for prognostic prediction of NPC patients.

In conclusion, the present data firstly provide a correlation between MIF and DJ-1 expression in NPCs. On the one hand, MIF and DJ-1 showed higher expression in tumor cells than that in normal nasopharyngeal lining epithelium, both high expression of MIF and DJ-1 promote cell invasion and migration in vitro, and play important role in contributing to lymph node metastasis in NPC. On the other hand, only MIF, but not DJ-1, is able to be a valuable prognostic factor to predict the patients' prognosis. Currently, clinical stage alone sometimes is inadequate to precisely predict the patients' prognosis. These two cell invasion-related molecules could help clinicians improve the prognostic prediction. From this standpoint, diminishing the expression of MIF or DJ-1 in tumor appears to be a promising strategy for developing new therapeutic approaches to treat this malignant tumor.

\section{Abbreviations}

MIF, macrophage migration inhibitory factor; NPC, nasopharyngeal carcinoma

\section{Acknowledgement}

This study is supported by Project of Guangdong Provincial Science and Technology (Grant number: 2012A030400008) and Project of Huizhou Municipal Central Hospital.

\section{Ethics Committee Approval and Patient Consent}

This study has been approved by Sun Yat-sen University and Huizhou Municipal Central Hospital review board or ethics committee.

\section{Competing Interests}

The authors have declared that no competing interest exists.

\section{References}

1. Yu MC, Yuan JM. Epidemiology of nasopharyngeal carcinoma. Semin Cancer Biol. 2002; 12:421-9.

2. Razak AR, Siu LL, Liu FF, et al. Nasopharyngeal carcinoma: the next challenges. Eur J Cancer. 2010; 46:1967-78.

3. El-Sherbieny E, Rashwan H, Lubis SH, et al. Prognostic factors in patients with nasopharyngeal carcinoma treated in Hospital Kuala Lumpur. Asian Pac J Cancer Prev. 2011; 12:1739-43.

4. O'Sullivan B. Nasopharynx cancer: therapeutic value of chemoradiotherapy. Int J Radiat Oncol Biol Phys.2007; 69:S118-21.

5. Farias TP, Dias FL, Lima RA, et al. Prognostic factors and outcome for nasopharyngeal carcinoma. Arch Otolaryngol Head Neck Surg. 2003; 129:794-9.

6. Pua KC, Khoo AS, Yap YY, et al. Nasopharyngeal Carcinoma Database. Med J Malaysia. 2008; 63 (Suppl C): 59-62.
7. Bach JP, Rinn B, Meyer B, et al. Role of MIF in inflammation and tumorigenesis. Oncology. 2008; 75:127-33.

8. Wang $\mathrm{XB}$, Tian $\mathrm{XY}, \mathrm{Li} \mathrm{Y}$, et al. Elevated expression of macrophage migration inhibitory factor correlates with tumor recurrence and poor prognosis of patients with gliomas. J Neurooncol. 2012; 106:43-51.

9. Lee H, Rhee H, Kang HJ, et al. Macrophage migration inhibitory factor may be used as an early diagnostic marker in colorectal carcinomas. Am J Clin Pathol. 2008; 129:772-9.

10. Ren Y, Tsui HT, Poon RT, et al. Macrophage migration inhibitory factor: roles in regulating tumor cell migration and expression of angiogenic factors in hepatocellular carcinoma. Int J Cancer. 2003; 107:22-9.

11. Chen $Y C$, Zhang XW, Niu XH, et al. Macrophage migration inhibitory factor is a direct target of HBP1-mediated transcriptional repression that is overexpressed in prostate cancer. Oncogene. 2010; 29:3067-78.

12. $\mathrm{Xu} \mathrm{X,} \mathrm{Wang} \mathrm{B}, \mathrm{Ye} \mathrm{C}$, et al. Overexpression of macrophage migration inhibitory factor induces angiogenesis in human breast cancer. Cancer Lett. 2008; 261:147-57.

13. Shimizu T, Abe R, Nakamura H, et al. High expression of macrophage migration inhibitory factor in human melanoma cells and its role in tumor cell growth and angiogenesis. Biochem Biophys Res Commun. 1999; 264:751-8.

14. Rendon BE, Roger $\mathrm{T}$, Teneng I, et al. Regulation of human lung adenocarcinoma cell migration and invasion by macrophage migration inhibitory factor. J Biol Chem. 2007; 282:29910-8.

15. Ogawa $\mathrm{H}$, Nishihira J, Sato $\mathrm{Y}$, et al. An antibody for macrophage migration inhibitory factor suppresses tumour growth and inhibits tumour-associated angiogenesis. Cytokine. 2000; 12:309-14.

16. Takahashi N, Nishihira J, Sato Y, et al. Involvement of macrophage migration inhibitory factor (MIF) in the mechanism of tumor cell growth. Mol Med. 1998, 4:707-14.

17. Bach JP, Deuster O, Balzer-Geldsetzer M, et al. The role of macrophage inhibitory factor in tumorigenesis and central nervous system tumors. Cancer. 2009; 115:2031-40.

18. Lue H, Thiele M, Franz J, et al. Macrophage migration inhibitory factor (MIF) promotes cell survival by activation of the Akt pathway and role for CSN5/JAB1 in the control of autocrine MIF activity. Oncogene. 2007; 26:5046-59.

19. Li GQ, Xie J, Lei XY, et al. Macrophage migration inhibitory factor regulates proliferation of gastric cancer cells via the PI3K/Akt pathway. World J Gastroenterol. 2009; 15:5541-8.

20. Lee CY, Su MJ, Huang CY, et al. Macrophage migration inhibitory factor increases cell motility and up-regulates av $\beta 3$ integrin in human chondrosarcoma cells. J Cell Biochem. 2012; 113:1590-8.

21. Li Z, Ren $Y, W u$ QC, et al. Macrophage migration inhibitory factor enhances neoplastic cell invasion by inducing the expression of matrix metalloproteinase 9 and interleukin-8 in nasopharyngeal carcinoma cell lines. Chin Med J (Engl). 2004; 117:107-14.

22. Liao B, Zhong BL, Li Z, et al. Macrophage migration inhibitory factor contributes angiogenesis by up-regulating IL-8 and correlates with poor prognosis of patients with primary nasopharyngeal carcinoma. J Surg Oncol. 2010; 102:844-51.

23. Nagakubo D, Taira T, Kitaura H, et al. DJ-1, a novel oncogene which transforms mouse NIH3T3 cells in cooperation with ras. Biochem Biophys Res Commun. 1997; 231:509-13.

24. Bonifati V, Rizzu P, van Baren MJ, et al. Mutations in the DJ-1 gene associated with autosomal recessive early-onset parkinsonism. Science. 2003; 299:256-9.

25. Davidson B, Hadar R, Schlossberg A, et al. Expression and clinical role of DJ-1, a negative regulator of PTEN, in ovarian carcinoma. Hum Pathol. 2008; 39:87-95.

26. Bindukumar B, Schwartz S, Aalinkeel R, et al. Proteomic profiling of the effect of prostate-specific antigen on prostate cancer cells. Prostate. 2008; 68:1531-45.

27. Tsuchiya B, Iwaya K, Kohno N, et al. Clinical significance of DJ-1 as a secretory molecule: retrospective study of DJ-1 expression at mRNA and protein levels in ductal carcinoma of the breast. Histopathology. 2012; 61:69-77.

28. He X, Zheng Z, Li J, et al. DJ-1 promotes invasion and metastasis of pancreatic cancer cells by activating SRC/ERK/uPA. Carcinogenesis. 2012; 33:555-62

29. Bai J, Guo C, Sun W, et al. DJ-1 may contribute to metastasis of non-small cell lung cancer. Mol Biol Rep. 2012; 39:2697-703.

30. Zhu XL, Wang ZF, Lei WB, et al. DJ-1: a novel independent prognostic marker for survival in glottic squamous cell carcinoma. Cancer Sci. 2010; 101:1320-5.

31. Min $\mathrm{H}$, Hong $\mathrm{M}, \mathrm{Ma} \mathrm{J}$, et al. A new staging system for nasopharyngeal carcinoma in China. Int J Radiat Oncol Biol Phys. 1994; 30:1037-42.

32. Kallioniemi OP, Wagner U, Kononen J, et al. Tissue microarray technology for high-throughput molecular profiling of cancer. Hum Mol Genet. 2001; 10:657-62.

33. Xie LQ, Bian LJ, Li Z, et al. Altered expression of E-cadherin by hepatocyte growth factor and effect on the prognosis of nasopharyngeal carcinoma. Ann Surg Oncol. 2010; 17:1927-36.

34. Hira E, Ono T, Dhar DK, et al. Overexpression of macrophage migration inhibitory factor induces angiogenesis and deteriorates prognosis after radical resection for hepatocellular carcinoma. Cancer. 2005; 103:588-98.

35. Legendre H, Decaestecker C, Nagy N, et al. Prognostic values of galectin-3 and the macrophage migration inhibitory factor (MIF) in human colorectal cancers. Mod Pathol. 2003; 16:491-504. 
36. Muramaki M, Miyake H, Yamada Y, et al. Clinical utility of serum macrophage migration inhibitory factor in men with prostate cancer as a novel biomarker of detection and disease progression. Oncol Rep. 2006; 15:253-7.

37. Leber MF, Efferth T. Molecular principles of cancer invasion and metastasis (review). Int J Oncol. 2009; 34:881-95.

38. Kraljevic Pavelic S, Sedic M, et al. Metastasis: new perspectives on an old problem. Mol Cancer. 2011; 10:22.

39. Ren $Y$, Law S, Huang $X$, et al. Macrophage migration inhibitory factor stimulates angiogenic factor expression and correlates with differentiation and lymph node status in patients with esophageal squamous cell carcinoma. Ann Surg. 2005; 242:55-63.

40. Vasseur S, Afzal S, Tardivel-Lacombe J, et al. DJ-1/PARK7 is an important mediator of hypoxia-induced cellular responses. Proc Natl Acad Sci USA. 2009; 106:1111-6.

41. He XY, Liu BY, Yao WY, et al. Serum DJ-1 as a diagnostic marker and prognostic factor for pancreatic cancer. J Dig Dis. 2011; 12:131-7.

42. Le Naour F, Misek DE, Krause MC, et al. Proteomics-based identification of RS/DJ-1 as a novel circulating tumor antigen in breast cancer. Clin Cancer Res. 2001; 7:3328-35.

43. Yuen HF, Chan YP, Law S, et al. DJ-1 could predict worse prognosis in esophageal squamous cell carcinoma. Cancer Epidemiol Biomarkers Prev. 2008; 17:3593-602.

44. Liu S, Yang Z, Wei H, et al. Increased DJ-1 and its prognostic significance in hepatocellular carcinoma. Hepatogastroenterology. 2010; 57:1247-56.

45. Fang M, Zhong XY, Du B, et al. Role of DJ-1-induced PTEN down-regulation in migration and invasion of human glioma cells. Chin J Cancer. 2010; 29:988-94.

46. Aleyasin H, Rousseaux MW, Marcogliese PC, et al. DJ-1 protects the nigrostriatal axis from the neurotoxin MPTP by modulation of the AKT pathway. Proc Natl Acad Sci USA. 2010; 107:3186-91.

47. Yang $Y$, Gehrke S, Haque ME, et al. Inactivation of Drosophila DJ-1 leads to impairments of oxidative stress response and phosphatidylinositol 3-kinase/Akt signaling. Proc Natl Acad Sci USA. 2005; 102:13670-5. 